\title{
Mercury Methylation by Cobalt Corrinoids: Relativistic Effects Dictate
} the Reaction Mechanism

\author{
Taye B. Demissie, Brady D. Garabato, Kenneth Ruud, and Pawel M. Kozlowski*
}

\begin{abstract}
The methylation of $\mathrm{Hg}^{\mathrm{II}}\left(\mathrm{SCH}_{3}\right)_{2}$ by corrinoid-based methyl donors proceeds in a concerted manner through a single transition state by transfer of a methyl radical, in contrast to previously proposed reaction mechanisms. This reaction mechanism is a consequence of relativistic effects that lower the energies of the mercury $6 p_{1 / 2}$ and $6 p_{3 / 2}$ orbitals, making them energetically accessible for chemical bonding. In the absence of spin-orbit coupling, the predicted reaction mechanism is qualitatively different. This is the first example of relativity being decisive for the nature of an observed enzymatic reaction mechanism.
\end{abstract}

Methylation is the process of controlled transfer of a methyl group onto amino acids, proteins, enzymes, DNA, or other biological intermediates. This process is an essential metabolic function and is catalyzed by a variety of enzymes, including corrinoid-dependent methyltransferases (MeTrs) $\cdot{ }^{[1-3]}$ One of the most well-studied MeTrs is cobalamin-dependent methionine synthase, which catalyzes the transfer of the methyl group of $\mathrm{CH}_{3}-\mathrm{H}_{4}$ folate to homocysteine to form methionine. ${ }^{[4,5]}$ Other MeTrs, such as CoFeSP, a module in the acetyl-CoA synthase, ${ }^{[5-7]}$ and methanol:coenzyme $\mathrm{M}$ have also been well studied. ${ }^{[8]}$

Cobalt corrinoids (Figure 1a) can formally transfer a methyl group in the form of a carbocation $\left(\mathrm{CH}_{3}^{+}\right)$, a radical $\left(\mathrm{CH}_{3}{ }^{\circ}\right)$, or a carbanion $\left(\mathrm{CH}_{3}{ }^{-}\right)$. Individual methyl transfer reactions have traditionally been interpreted as $\mathrm{S}_{\mathrm{N}} 2$-type displacements (i.e., formally involving $\left.\mathrm{CH}_{3}{ }^{+}\right) \cdot{ }^{[2-4]}$ Alternative mechanisms based on reductive cleavage (or one-electron reduction) of the corrinoid induced by certain substrates have also been proposed. ${ }^{[9-12]}$ The key step in this reductive cleavage mechanism involves formation of a diradical species, which pre-activates the reactant complex and facilitates homolytic cleavage of the $\mathrm{Co}-\mathrm{C}$ bond followed by $\mathrm{CH}_{3}{ }^{\circ}$

[*] Dr. T. B. Demissie, Prof. Dr. K. Ruud

Centre for Theoretical and Computational Chemistry

Department of Chemistry

UiT The Arctic University of Norway

9037 Tromsø (Norway)

B. D. Garabato, Prof. Dr. P. M. Kozlowski

Department of Chemistry, University of Louisville

2320 South Brook Street, Louisville, KY 40292 (USA)

E-mail:pawel@louisville.edu

Prof. Dr. P. M. Kozlowski

Visiting Professor, Department of Food Sciences

Medical University of Gdansk

Al. Gen. J. Hallera 107, 80-416 Gdansk (Poland)

(iD)

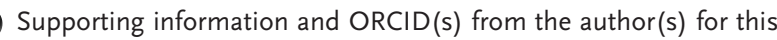

article are available on the WWW under:

http://dx.doi.org/10.1002/anie.201606001. a)
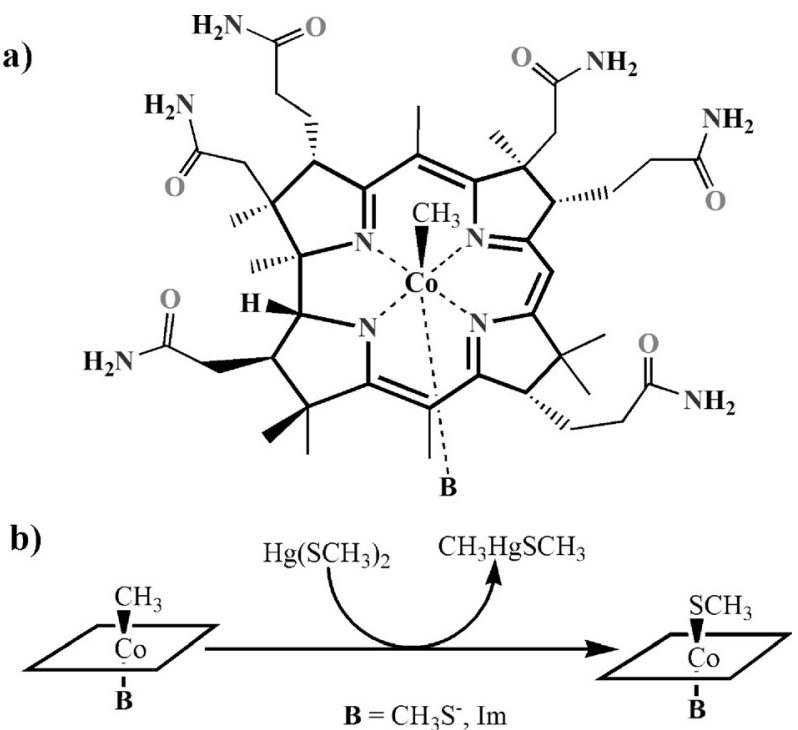

Figure 1. a) Molecular structure of the cobalt corrinoid. b) Ligand exchange reaction between $\mathrm{Hg}^{\prime \prime}\left(\mathrm{SCH}_{3}\right)_{2}$ and $\mathbf{B}-[\mathrm{Co}$ "II corrin $]-\mathrm{CH}_{3}{ }^{+}$.

transfer. Until recently, there had been no evidence for a methyl anion transfer, which would result in a $\mathrm{Co}^{\mathrm{III}}$ product (or intermediate) after $\mathrm{Co}-\mathrm{C}$ bond heterolysis: However, the involvement of $\mathrm{CH}_{3}{ }^{-}$has been invoked in the context of $\mathrm{Hg}^{\mathrm{II}}$ methylation, specifically in conjunction with bacterial mercury methylation $(\mathrm{HgcA})$ from $D$. desulfuricans ND132. The feasibility of $\mathrm{CH}_{3}{ }^{-}$involvement in methyl transfer reactions was further supported by density functional theory (DFT) calculations of ligand exchange reactions (LERs) between cobalt corrinoids and $\mathrm{Hg}^{\mathrm{II}}$-based substrates. ${ }^{[13]}$

Herein, we demonstrate that spin-orbit effects are crucial for a proper description of LERs between $\mathrm{Hg}^{\mathrm{II}}$ and corrinoidbased methyl donors (Figure $1 \mathrm{~b}$ ). It is shown that the inclusion of spin-orbit coupling (SOC) effects changes the mechanism of the LER, which becomes more consistent with radical methyl transfer, rather than involving a methyl carbanion. Furthermore, the presence of SOC changes the nature of the reaction pathway, from a stepwise to a concerted LER between $\mathrm{Co}^{\mathrm{III}}$ and $\mathrm{Hg}^{\mathrm{II}}$.

In this study, we analyzed the LER of $\mathrm{Hg}^{\mathrm{II}}\left(\mathrm{SCH}_{3}\right)_{2}$ methylation by $\mathbf{B}-\left[\mathrm{Co}^{\mathrm{III}}\right.$ corrin $]-\mathrm{CH}_{3}^{+}$(Figure $1 \mathrm{~b}$ ), which results in $\mathrm{CH}_{3} \mathrm{Hg}^{\mathrm{II}} \mathrm{SCH}_{3}$ and $\mathbf{B}-\left[\mathrm{Co}^{\mathrm{III}}\right.$ corrin $]-\mathrm{SCH}_{3}{ }^{+}$. As in previous computational studies, ${ }^{[14,15]}$ a truncated structure of the corrin was used (Figure 1a). To obtain a reliable description of the LER, relativistic BP86-D3/DFT calculations employing the zeroth-order regular approximation (ZORA) to the Dirac equation were applied. In addition, SOC was 
included in the ZORA Hamiltonian (SO-ZORA), and relativistic basis sets for all atoms were used. Environmental effects were modelled through $\mathrm{COSMO} / \mathrm{H}_{2} \mathrm{O}$ as implemented in the ADF suite of programs for electronic-structure calculations (see the Supporting Information for details). ${ }^{[6]}$

Potential energy surfaces (PESs) for the LERs computed at the SO-ZORA/BP86-D3/TZ2P $\left(\mathrm{COSMO} / \mathrm{H}_{2} \mathrm{O}\right)$ level of theory are shown in Figure $2 \mathrm{a}$ and Figure S1 of the Supporting Information, with imidazole ( Im) and cysteine (Cys) as the lower axial ligands coordinated to $\mathrm{Co}^{\mathrm{III}}(\mathbf{B})$, respectively.
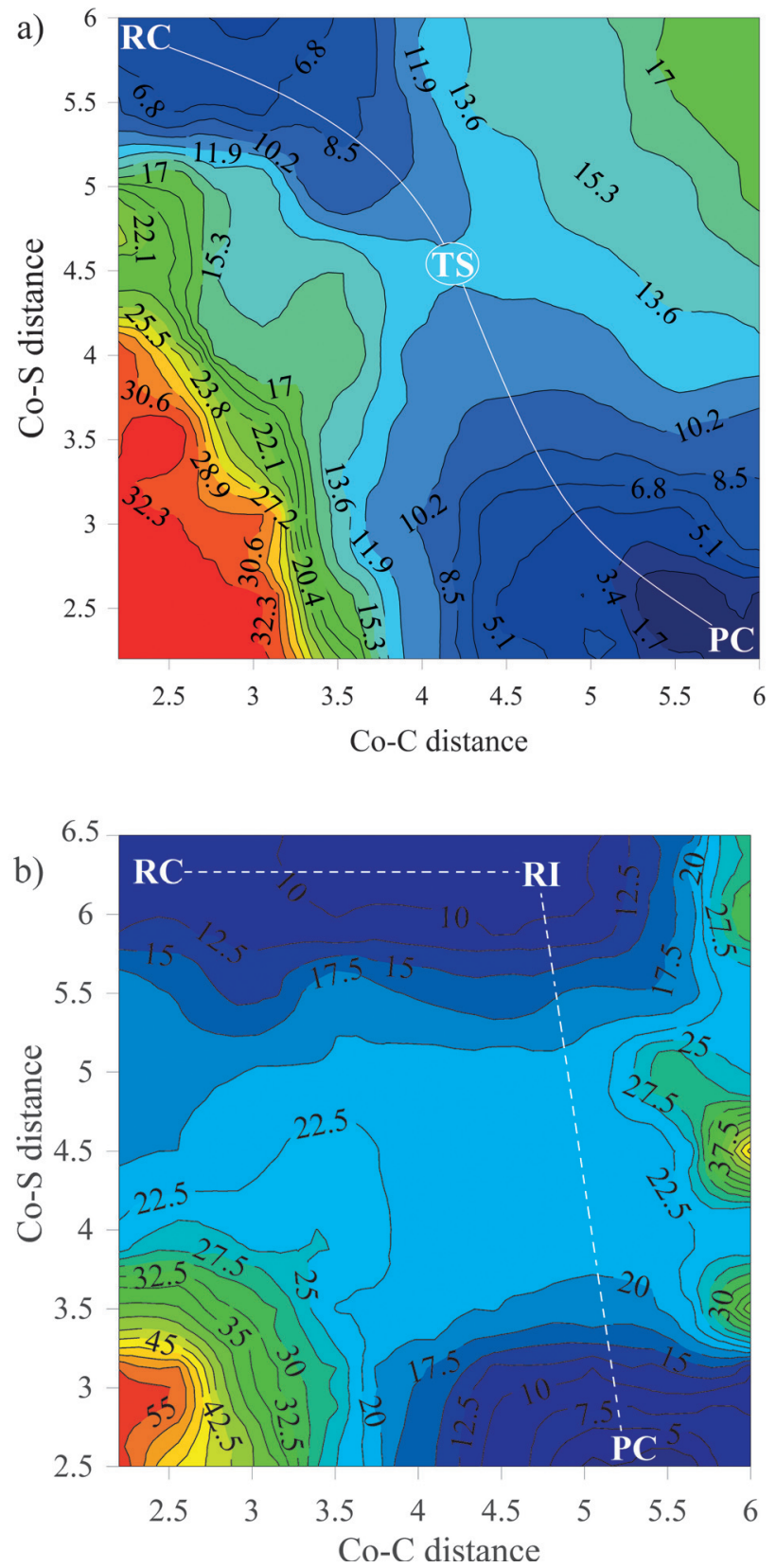

Figure 2. a) $P E S$ corresponding to the $L E R$ with $\mathbf{B}=I m$ calculated at the SO-ZORA/BP86-D3/TZ2P (COSMO/ $\left.\mathrm{H}_{2} \mathrm{O}\right)$ level of theory. b) PES corresponding to the LER with $\mathbf{B}=\mathrm{Im}$ calculated at the NR/BP86-D3/ TZ2P $\left(\mathrm{COSMO} / \mathrm{H}_{2} \mathrm{O}\right)$ level of theory.
Whereas nitrogenous trans ligands, such as dimethylbenzimidazole (DBI) and histidine (His), are commonly found in $\mathrm{B}_{12}$-dependent enzymes, in the case of $\mathrm{HgcA}$, a strictly conserved Cys residue was predicted to be the lower $\mathrm{Co}^{\text {III }}$ coordinated ligand. ${ }^{[17]}$ It was further argued that the presence of a Cys axial base in $\mathrm{HgcA}$ enhances the methylation of $\mathrm{Hg}^{\mathrm{II}}$. Both PESs were constructed as functions of the $\mathrm{Co}-\mathrm{C}$ and $\mathrm{Co}-\mathrm{S}$ distances, and although other internal coordinates were tested, the most transparent description of the LERs was provided by PESs with coordinates associated with the $\mathrm{Co}^{\mathrm{III}}$ center (Figure 2). Overall, regardless of the axial base, the SO-ZORA DFT calculations gave very consistent descriptions of the mechanisms associated with the LERs (see Figure $2 \mathrm{a}$ and Figure S1). The minimum-energy reaction pathway connecting the reactant complex (RC) and the product complex (PC) proceeds via a single transition state (TS). DFT calculations that included SOC effects clearly predicted the ligand exchange to be concerted, rather than stepwise, in contrast to previous suggestions. ${ }^{[13]}$

We then considered the LERs between $\mathrm{Co}^{\mathrm{III}}$ and $\mathrm{Hg}^{\mathrm{II}}$ without relativistic effects. Using the same structural models and basis sets, a nonrelativistic (NR) PES was constructed based on BP86-D3/TZ2P $\left(\mathrm{COSMO} / \mathrm{H}_{2} \mathrm{O}\right)$ calculations (Figure $2 \mathrm{~b}$; note that only one surface is shown with $\mathbf{B}=\mathrm{Im}$, as different axial bases produce qualitatively similar PESs). The NR PES looks very different from the corresponding PES shown in Figure $2 \mathrm{a}$. Starting from the RC, a minimum-energy path lies parallel to the $\mathrm{Co}^{-} \mathrm{C}$ coordinate, implying that the initial step involves cleavage of one bond without formation of the other. The energy minimum corresponding to the reaction intermediate (RI) involves formation of $\mathrm{CH}_{3} \mathrm{~S}^{-}$and $\mathrm{CH}_{3} \mathrm{Hg}^{\mathrm{II}} \mathrm{SCH}_{3}$. Furthermore, the presence of a $\mathrm{Co}^{\mathrm{III}}$-based corrinoid indicates that the $\mathrm{Co}-\mathrm{C}$ bond is cleaved heterolytically, and that the methyl group is formally transferred as $\mathrm{CH}_{3}{ }^{-}$in the LER. ${ }^{[13]}$ The absence of relativistic effects thus leads to a very different mechanistic picture of the LER, which now proceed in a stepwise manner and involves $\mathrm{CH}_{3}{ }^{-}$.

To further explore the nature of the LERs, the corresponding TSs were located and characterized taking relativistic effects into account. For compatibility with the PES shown in Figure $2 \mathrm{a}$, the corresponding energy profiles were computed with respect to the RC (Figure 3). Figure 3 also gives spin density profiles as well as key structural parameters. Regardless of the axial base used in the SO-ZORA/BP86-D3/ TZ2P $\left(\mathrm{COSMO} / \mathrm{H}_{2} \mathrm{O}\right)$ calculations, the predicted LERs are consistent with radical mechanisms. This conclusion is supported by an analysis of the spin density profiles, which show that the unpaired electrons are located either on Co and $\mathrm{C}$ or on $\mathrm{S}$ depending on the axial base, which is consistent with the open-shell diradical character of the TSs. The spin density associated with the cobalt center has a $\mathrm{d}_{\mathrm{z}^{2}}$ orbital shape, indicating $\mathrm{Co}^{\mathrm{II}}$ character and further supporting the hypothesis that the LERs involve the transfer of a methyl radical.

The key difference between the LERs with Cys or Im as the axial base is the energy difference between the RC and the TS, which is almost twice as large in the case of Im. These differences in energy can be attributed to the trans effect associated with the axial base, as can be inferred from the spin density profiles associated with the Im and Cys ligands 


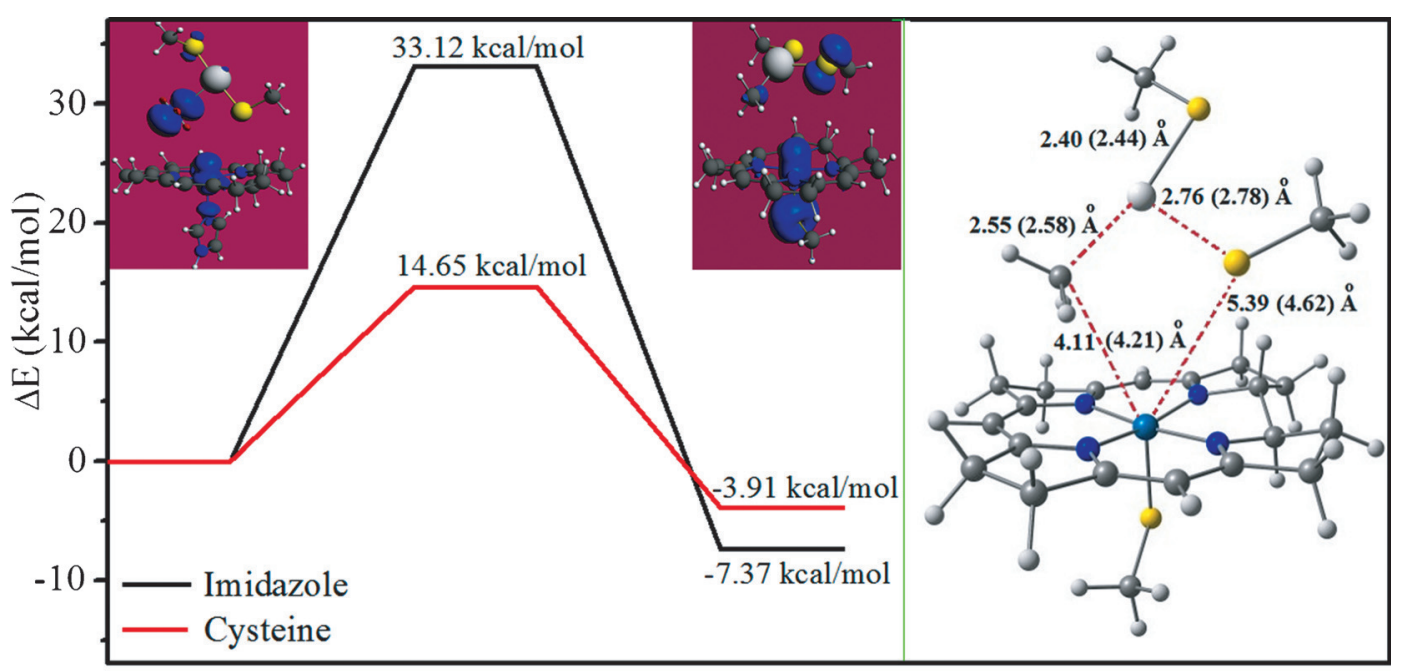

Reaction coordinate

Figure 3. Reaction profiles of the LER of $\mathrm{Hg}^{\prime \prime}\left(\mathrm{SCH}_{3}\right)_{2}$ methylation by $\mathbf{B}-[\mathrm{Co}$ 'I'corrin $]-\mathrm{CH}_{3}{ }^{+}$for $\mathbf{B}=$ Im and Cys along with the spin densities in the transition states for $\mathbf{B}=\operatorname{Im}$ (left) and $\mathbf{B}=$ Cys (middle). The transition state for $\mathbf{B}=$ Cys is shown on the right where also important distances for $\mathrm{B}=\mathrm{Im}$ are given in parentheses.

(Figure 3). In the case of Im, the majority of the spin is located on $\mathrm{Co}$ and $\mathrm{C}_{\mathrm{Me}}$, with only a small contribution from $\mathrm{N}_{\mathrm{Im}}$. The corresponding TS can thus be described as an early TS. On the other hand, the spin density distribution looks quite different for Cys. The spin density is largely shared between $\mathrm{Co}^{\mathrm{II}}$ and $\mathrm{S}_{\mathrm{Hg}}$, but with a non-negligible contribution from $\mathrm{S}_{\mathrm{Cys}}$. In contrast to the Im scenario, the corresponding TS can be viewed as a late TS, which is closer to the PC than to the RC. The ability of $\mathrm{S}_{\mathrm{Cys}}$ to accommodate more electron density than $\mathrm{N}_{\text {Im }}$ leads to a stabilization of the TS by shifting the spin density associated with the upper ligand from $\mathrm{C}_{\mathrm{Me}}$ to $\mathrm{S}_{\mathrm{Me}}$.

The mechanism for the reaction with Cys as the trans axial base was further investigated with an analysis of the eigenvector associated with the imaginary frequency $i 116 \mathrm{~cm}^{-1}$. The TS can be described as having no motion along the $\mathrm{Co}-\mathrm{C}$ coordinate, which is consistent with a distance between $\mathrm{Co}$ and $\mathrm{C}_{\mathrm{Me}}$ in the TS that is equal to $4.11 \AA$, compared to an equilibrium $\mathrm{Co}-\mathrm{C}$ bond length of $2.00 \AA$. Likewise, the distance between $\mathrm{C}$ and $\mathrm{S}_{\mathrm{Me}}$ of $5.39 \AA$ is also long. However, the concerted nature of the reaction, in which the $\mathrm{CH}_{3}$ group is partially transferred to $\mathrm{Hg}^{\mathrm{II}}$ while the $\mathrm{SCH}_{3}$ group is still weakly bound, should be favored by the presence of cob(II)alamin. This observation is in agreement with the hypothesis that cob(II)alamin acts as a conductor that is involved in the stabilization of highly reactive product-related methylene radicals in $\mathrm{B}_{12}$-dependent enzymatic catalysis. ${ }^{[18]}$

It is interesting to understand why the LER mechanism obtained without taking relativistic effects into account is so different. The reason can be found in the electronic structure of $\mathrm{Hg}^{\mathrm{II}}$. When relativistic effects are included, the relative energy levels of the atomic orbitals change, and subshells with $l>0$ split into two subgroups ("spinors") because of SOC. ${ }^{[19,20]}$ Specifically, the $6 \mathrm{~s}$ orbitals of $\mathrm{Hg}$ substantially decrease in energy while the $6 \mathrm{p}$ orbitals decrease in energy to a lesser extent. The $6 p$ subshell splits into $6 p_{1 / 2}$ and $6 p_{3 / 2}$ spinors. The energetic accessibility of the $6 \mathrm{p}$ orbitals is clearly enhanced in the coordination sphere of $\mathrm{Hg}^{\mathrm{II}}$ when the structure of the TS is taken into consideration (see Figures S2 and S3). The ability of mercury to coordinate three ligands, namely $\mathrm{CH}_{3}$ and two $\mathrm{SCH}_{3}$ groups in a T-shaped complex (Figure 3), is consistent with mixing of the $6 \mathrm{~s}$ and two $6 \mathrm{p}$ orbitals. The frontier orbitals of the corresponding TS are presented in Figures S4-S7. Without SOC, the $6 \mathrm{p}$ orbitals are not energetically accessible and cannot participate in chemical bonding. Consequently, $\mathrm{Hg}^{\mathrm{II}}$ cannot accommodate three ligands, and $\mathrm{SCH}_{3}{ }^{-}$is released as an intermediate to enable coordination of the methyl group to mercury in the non-relativistic regime.

Finally, it should be mentioned that relativistic effects are crucial for describing the properties of elements such as gold and mercury ${ }^{[19-22]}$ and for explaining the electronic configuration of neutral atoms of the $\mathrm{d}$ and $\mathrm{f}$ block. ${ }^{[23]}$ It has also been demonstrated that scalar relativistic effects enhance the catalytic activity of gold. ${ }^{[24]}$ Herein, we have shown for the first time that spin-orbit effects are essential for describing a heavy-metal-involving enzymatic reaction. Theory only supports a carbanion transfer to $\mathrm{Hg}^{\mathrm{II}}$ when SOC is omitted. ${ }^{[13]}$ When spin-orbit effects are taken into account, the TS has a multicentered diradical character, and the methyl group is transferred in the form of a radical.

\section{Acknowledgements}

This work was supported by the Research Council of Norway through a Centre of Excellence grant (179568/V30) and a research grant (177558/V00) as well as by the Norwegian Program for Supercomputing (NOTUR) through a computer time grant (nn4654k). This work was also funded by the National Science Centre, Poland (UMO-2015/17/B/ST4/ 03733). The Cardinal Research Cluster (Supercomputing Facilities at the University of Louisville) is acknowledged for providing computational resources. 
Keywords: computational chemistry .

density functional calculations · enzyme catalysis .

reaction mechanisms $\cdot$ transition states

How to cite: Angew. Chem. Int. Ed. 2016, 55, 11503-11506 Angew. Chem. 2016, 128, 11675-11678

[1] R. Banerjee, Chemistry and Biochemistry of B12, Wiley, New York, 1999.

[2] R. Banerjee, S. W. Ragsdale, Annu. Rev. Biochem. 2003, 72, 209.

[3] R. G. Matthews in Metal Ions in Life Sciences, Vol. 6 (Eds.: A. Sigel, H. Sigel, R. K. O. Sigel), Royal Society of Chemistry, 2009, pp. $53-114$.

[4] R. G. Matthews, Acc. Chem. Res. 2001, 34, 681.

[5] R. G. Matthews, M. Koutmos, S. Datta, Curr. Opin. Struct. Biol. 2008, 18, 658.

[6] T. Doukov, J. Seravalli, J. J. Stezowski, S. V. Ragsdale, Structure 2000, $8,817$.

[7] T. Svetlitchnaia, V. Svetlitchnyi, O. Meyer, H. Dobbek, Proc. Natl. Acad. Sci. USA 2006, 103, 14331.

[8] C. H. Hagemeier, M. Kr̈er, R. K. Thauer, E. Warkentin, U. Ermler, Proc. Natl. Acad. Sci. USA 2006, 103, 18917.

[9] P. M. Kozlowski, J. Kuta, W. Galezowski, J. Phys. Chem. B 2007, 111,7638 .

[10] M. Alfonso-Prieto, X. Blarnes, M. Kumar, C. Rovira, P. M. Kozlowski, J. Phys. Chem. B 2010, 114, 12965.

[11] N. Kumar, M. Jaworska, P. Lodowski, M. Kumar, P. M. Kozlowski, J. Phys. Chem. B 2011, 115, 6722.

[12] N. Kumar, P. M. Kozlowski, J. Phys. Chem. B 2013, 117, 16044.
[13] J. Zhou, D. Riccardi, A. Beste, J. C. Smith, J. M. Parks, Inorg. Chem. 2014, 53, 772.

[14] P. M. Kozlowski, M. Kumar, P. Piecuch, W. Li, N. P. Bauman, J. A. Hansen, P. Lodowski, M. Jaworska, J. Chem. Theory Comput. 2012, 8, 1870.

[15] T. B. Demissie, M. Repisky, H. Liu, K. Ruud, P. M. Kozlowski, J. Chem. Theory Comput. 2014, 10, 2125.

[16] ADF2014, SCM, Theoretical Chemistry, Vrije Universiteit Amsterdam, The Netherlands, http://www.scm.com/, 2014.

[17] S. D. Smith, R. Bridou, A. Johs, J. M. Parks, D. A. Elias, R. A. Hurt, Jr., S. D. Brown, M. Podar, J. D. Wall, Appl. Environ. Microbiol. 2015, 81, 3205.

[18] P. M. Kozlowski, T. Kamachi, T. Toraya, K. Yoshizawa, Angew. Chem. Int. Ed. 2007, 46, 980; Angew. Chem. 2007, 119, 998.

[19] P. Pyykko, Chem. Rev. 1988, 88, 563.

[20] J. S. Thayer, J. Chem. Educ. 2005, 82, 1721.

[21] F. Calvo, E. Pahl, M. Wormit, P. Schwerdtfeger, Angew. Chem. Int. Ed. 2013, 52, 7583; Angew. Chem. 2013, 125, 7731.

[22] P. Schwerdtfeger, P. D. W. Boyd, S. Brienne, J. S. McFeaters, M. Dolg, M.-S. Liao, W. H. E. Schwarz, Inorg. Chim. Acta 1993, 213, 233.

[23] J. Autschbach, S. Siekierski, M. Seth, P. Schwerdtfeger, W. H. E. Schwarz, J. Comput. Chem. 2002, 23, 804.

[24] M. Lein, M. Rudolph, S. K. Hashmi, P. Schwerdtfeger, Organometallics 2010, 29, 2206.

Received: June 21, 2016

Published online: August 11, 2016 